\title{
Nanofiber Filter Performance Improvement: Nanofiber Layer Uniformity and Branched Nanofiber
}

\author{
Seong Chan Kim ${ }^{1 *}$, Seungkoo Kang ${ }^{1}$, Handol Lee ${ }^{1}$, Dong-Bin Kwak ${ }^{1}$, Qisheng Ou ${ }^{1}$, \\ Chenxing Pei ${ }^{1}$, David Y.H. Pui ${ }^{1,2}$ \\ ${ }^{1}$ Department of Mechanical Engineering, University of Minnesota, Minneapolis, MN 55455, USA \\ ${ }^{2}$ School of Science and Engineering, The Chinese University of Hong Kong, Shenzhen, Guangdong 518172, China
}

\begin{abstract}
We developed two types of high performance nanofiber filters by increasing the uniformity of the fiber layer's deposition on the substrate media and by fabricating nanofiber with a branched morphology, in which additional fibers were spun to radiate from the main fibers. We subsequently compared them with conventional and beaded nanofiber filters in terms of particle removal efficiency, filter pressure drop, and particle loading capacity. First, the uniformity of the nanofiber's deposition, which was visually evaluated using optical and electron microscopes, was increased by removing the substrate surface charge during electrospinning. The uniform nanofiber filters demonstrated a higher filtration efficiency with a lower pressure drop than their conventionally electrospun counterparts, which exhibited irregular fiber layers. Second, branched nanofiber was fabricated by adjusting the viscosity of the polymer solution. The performance of the nanofiber filters was dramatically improved by the decrease in air flow resistance, which resulted from (1) a longer distance streamwise between the fibers and (2) a smaller diameter for the fibers. The enhanced efficiency was also due to (3) the additional fibers branching out from the main fibers.
\end{abstract}

Keywords: Nanofiber; Beaded nanofiber; Branched nanofiber; Uniformity; Figure of merit.

\section{INTRODUCTION}

In past decades, it has been well known that particulate matters pose a great threat to human health. Especially airborne particles smaller than $2.5 \mu \mathrm{m}\left(\mathrm{PM}_{2.5}\right)$ can easily penetrate the human respiratory system and translocate to secondary organs including the brain causing various severe health issues including lung disease, heart disease and even premature death (Nel, 2005; Stone and Donaldson, 2006; Oberdörster et al., 2007). Many efforts have been made to reduce human exposure to particulate matters and minimize its health effects. Among the various efforts to control the air quality, air filtration is the most cost-effective way to remove airborne particles from inlet air flow. However, as the concerned particle size gets smaller, air filter media needs to be denser and thicker to improve filtration efficiency, which results in increasing filter pressure drop and thus increasing total operation cost. In order to solve this problem, many researchers have investigated nanofiber filter media, which consists of nanometer-scale fibers layered on top of

\footnotetext{
${ }^{*}$ Corresponding author.

Tel.: 1-612-626-1517

E-mail address: sckim@umn.edu
}

a regular micrometer-scale filter media as a substrate, and these nanofiber filters improved filtration performance dramatically in terms of both filtration efficiency and pressure drop (Leung et al., 2009; Choi et al., 2017). Recently, beaded nanofiber filters have been proposed to decrease pressure drop because of larger streamwise inter-fiber distance and similar to the regular nanofiber filters performed with high filtration efficiency (Fong et al., 1999; Yun et al., 2010; Wang et al., 2015; Gao et al., 2017).

In the present research, in order to improve nanofiber filter performance, nanofiber layer uniformity was improved by removing substrate surface charge during the electrospinning process. This performance improvement was evaluated through visual inspections and filtration tests. Branched nanofiber filter media with numerous additional nanofibers spun out from the main nanofibers was developed to improve overall filtration performance. The filtration performance was compared with regular nanofiber filters and beaded nanofiber filters in terms of particle removal efficiency, filter pressure drop and particle loading capacity.

\section{METHODS}

\section{Electrospinning and Beaded Nanofiber}

Fig. 1(a) shows a typical electrospinning setup consisting of a syringe pump with a conductive needle, a high voltage 
supplier and a grounded collector. First, polymer powder is dissolved in solvent using a mechanical stirrer at ambient temperature to minimize any possible damage on polymer chain structure and the polymer solution is delivered through a conductive needle at a controlled feeding rate using a syringe pump. Then the polymer solution is electrically charged by a high voltage power supplier when passing through the conductive needle and spun out by an electrostatic force between the needle tip and a grounded collector. The collected polymer fiber has very uniform fiber diameter in nanometer ranges and the fiber diameter and shape can be controlled by polymer solution properties like viscosity, surface tension, conductivity as well as electrospinning conditions like solution feeding rate, needle size, and electric field strength (Drew et al., 2003; Huang et al., 2003; Shenoy et al., 2005; Hekmati et al., 2013). It is well known that the nanofiber diameter is proportional to its polymer solution viscosity, which means a smaller fiber diameter can be fabricated by using lower viscosity polymer solution. However, when the solution viscosity is lower than a critical value, which is decided by the balance between solution viscosity and surface tension, polymer beads are formed along nanofibers as shown in Fig. 1(b) (right) (Baumgarten, 1971; McKee et al., 2004). These beaded nanofibers have been considered as defective because these beads keep streamwise inter-fiber distance longer than smooth nanofibers (Fig. 1(b), left) causing filtration efficiency decrease. Recently, researchers have investigated to use beaded nanofibers as a good candidate for air filter media because this longer interfiber distance of beaded nanofibers can improve filtration performance by increasing air permeability while still providing high filtration efficiencies.

\section{Uniform Nanofiber Layer Deposition}

Since nanofiber layer itself does not have enough mechanical strength to be used as filter media, composite filter media are manufactured to form a thin nanofiber layer on top of a substrate filter media. With this combination of a nanofiber layer and a substrate filter, nanofiber filter presents high filtration efficiency with sufficient mechanical support. However, commercialized nanofiber filters often present lower filtration efficiencies than their design specification due to the non-uniformity of nanofiber layer deposition. This has previously been a well-known issue in the fabrication of nanofiber filters due to the non-uniform surface charge distribution on the substrate filter surface (Fig. 2(a), left). This is because the electric field between the conductive needle tip and the grounded substrate surface is non-uniformly distributed on the uneven substrate filter surface as shown in the Fig. 2(a) (right) (Barhate et al., 2006; Barhate and Ramakrishna, 2007; Wang et al., 2009; Xu et al., 2016). Fig. 2(b) shows SEM (scanning electron microscope) images of nanofiber deposition on cellulose filter substrate using conventional electrospinning setup. The image on the right (Fig. 2(b)) shows a highly dense nanofiber deposition along the substrate cellulose fiber causing high air resistance. However, in the area shown in Fig. 2(b) (left), the nanofiber layer is widely open and acts like "pinholes," thus causing a dramatic decrease of the filtration efficiency. This means the nanofiber layer non-uniformity plays a negative role in both

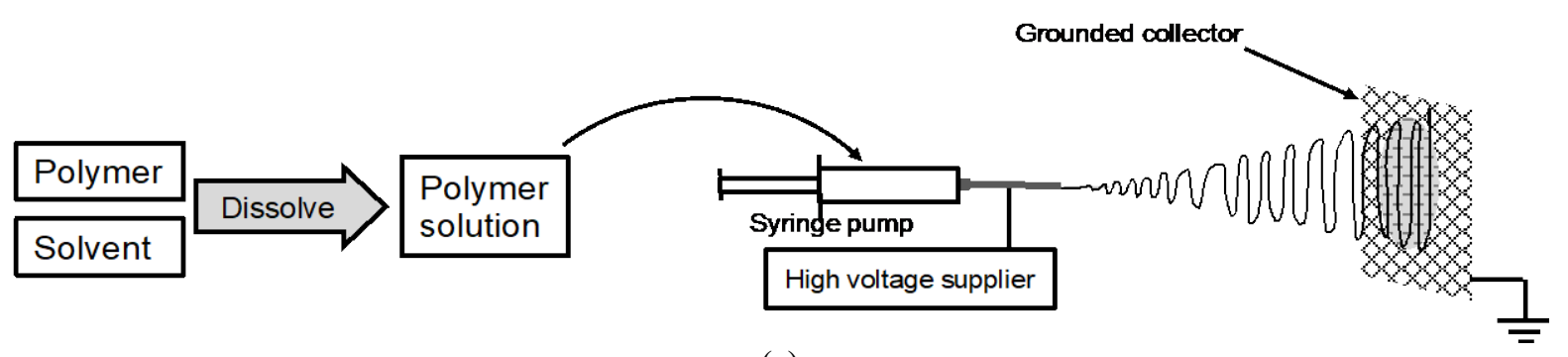

(a)
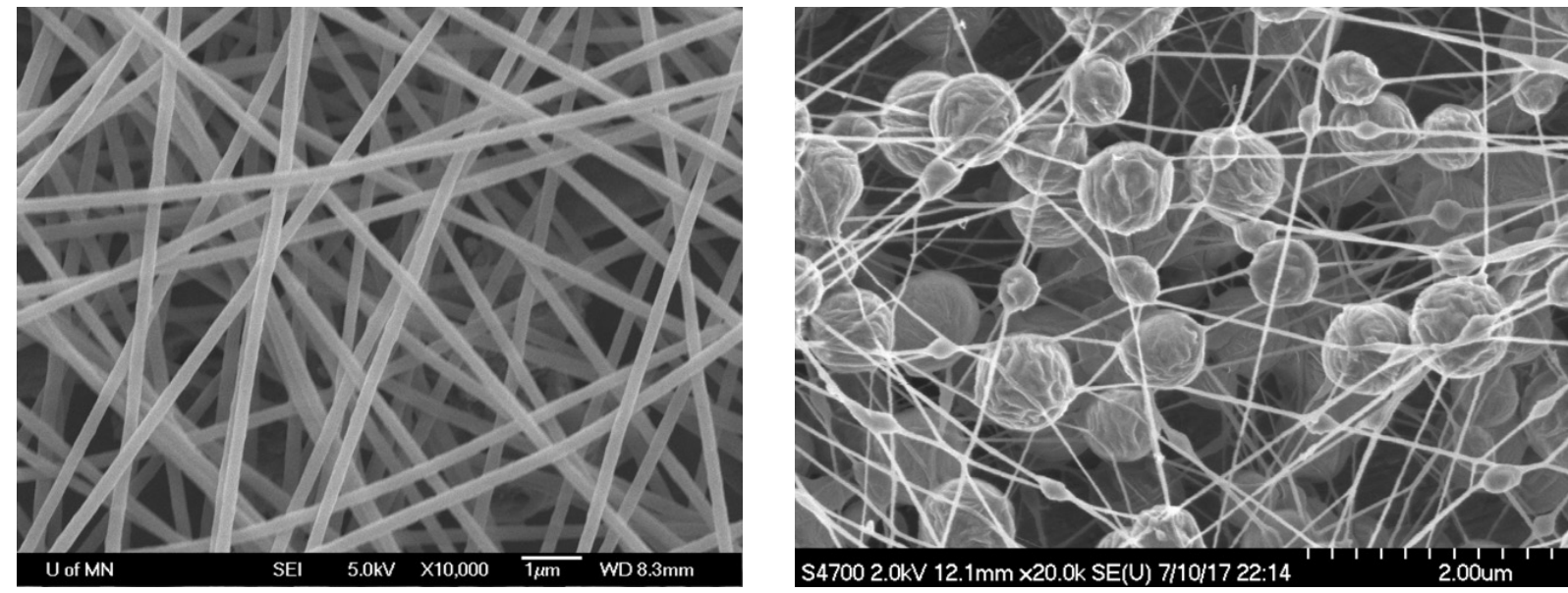

(b)

Fig. 1. (a) Basic concept of electrospinning method and (b) smooth nanofibers (left) and beaded nanofibers (right). 

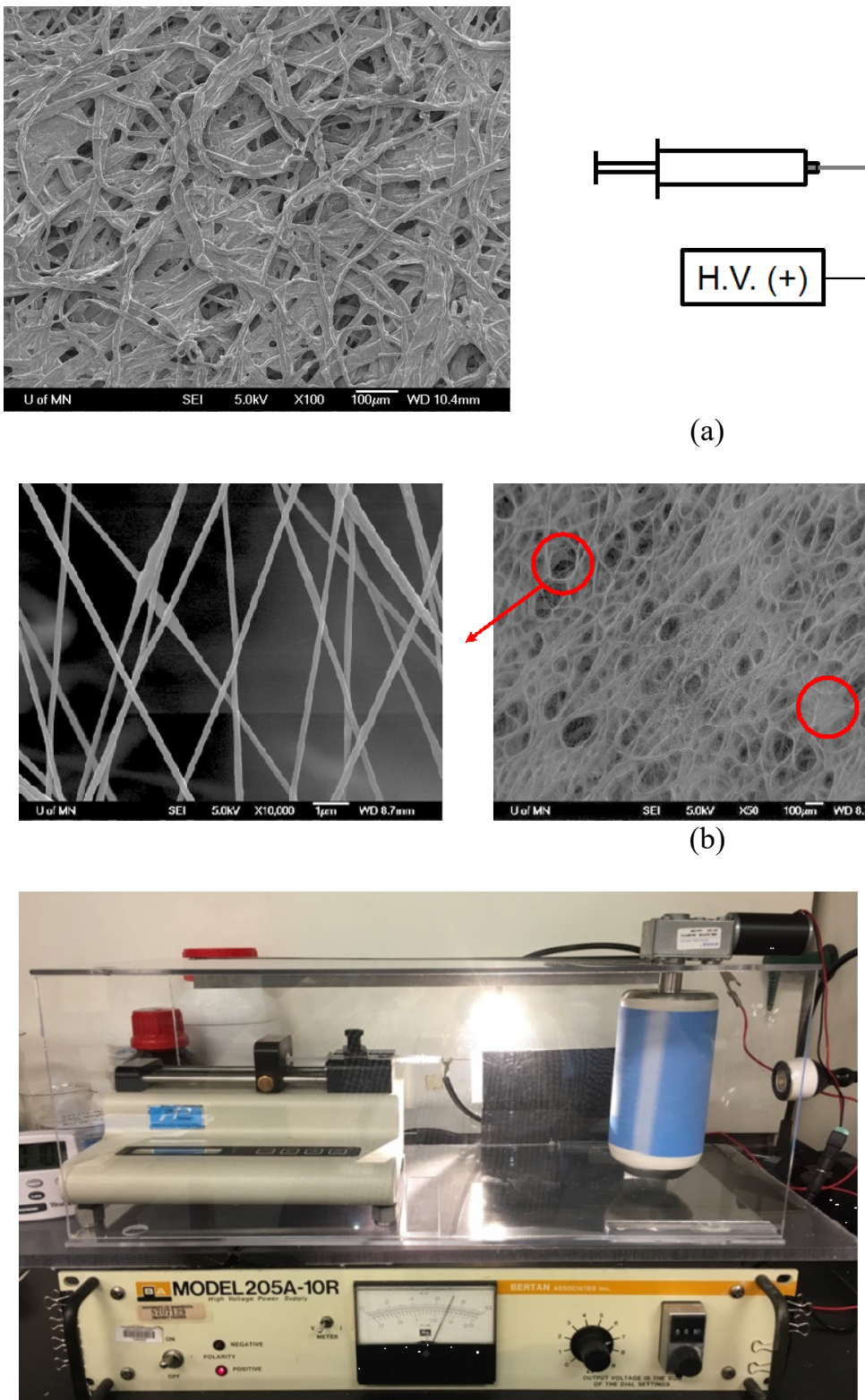

(c)

(b)

(a)
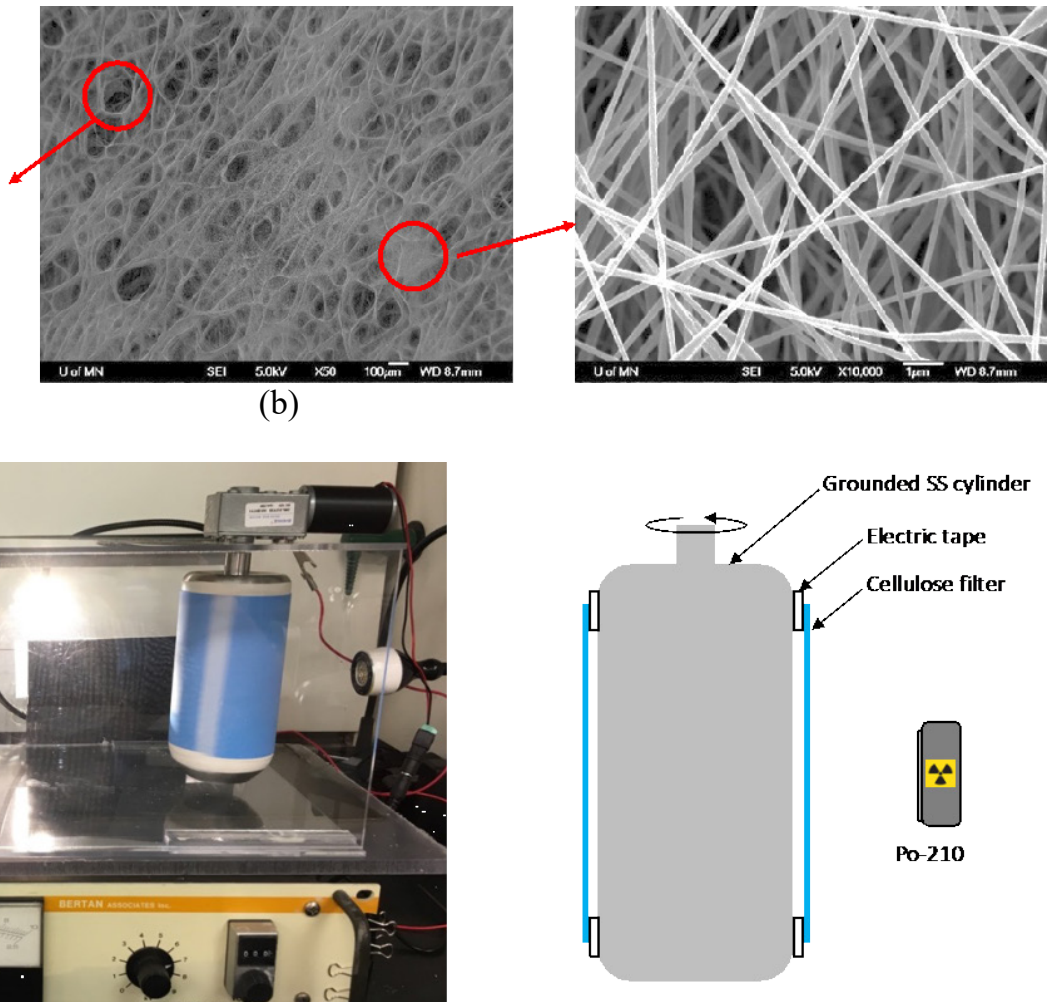

Fig. 2. Improvement of nanofiber layer uniformity: (a) non-uniform electric field on the substrate filter surface, (b) SEM images of non-uniform nanofiber filter, and (c) electrospinning setup to improve nanofiber layer uniformity.

the filtration efficiency and the filter pressure drop. Therefore, it is important to make uniform nanofiber deposition on the substrate filter media in order to improve the overall filtration performance. Fig. 2(c) shows the experimental electrospinning setup used to make a uniform nanofiber deposition, which consists of a syringe pump (EW-74900; Cole-Parmer), a $22 \mathrm{G}$ Blunt tip syringe, a high voltage power supply (205A10R; Bertan Inc.), and a grounded rotating cylinder (70 mm in diameter) with cellulose filter media wrapping around it. In this research, nanofibers were fabricated with fixed operating conditions, such as polymer solution feeding rate $\left(0.50 \mathrm{~mL} \mathrm{~h}^{-1}\right)$, applied voltage $(8.0 \mathrm{kV})$, needle tip-to-collector distance $(10 \mathrm{~cm})$ and cylinder rotating speed $(250 \mathrm{rpm})$.

As mentioned in the previous paragraph, in order to make a uniform nanofiber deposition on substrate filter media, we need to remove the non-uniform surface charge on the substrate filter as well as create a uniform electric field between the needle tip and the grounded cylinder. Therefore, the uniform nanofiber deposition can be created by (1) neutralizing the substrate filter surface using an ionizer in the opposite side of the electrospinning setup and (2) electrically isolating the substrate filter media from the grounded cylinder using electric tapes wrapped around the top and bottom of the cylinder. In this research, we used ${ }^{210} \mathrm{Po}$ radioactive source (P-2042, $185 \mathrm{MBq}$; NRD Inc.) as an ionizer. This ionizer diminished the surface charge on the substrate filter media and the electrospun nanofiber is transported along the uniform electric field between the needle tip and the smooth cylinder surface and deposited uniformly on the substrate filter surface. 
Fig. 3 compares 3 set of images between non-uniform (Fig. 3(a)) and uniform nanofiber deposition (Fig. 3(b)), photographs of nanofiber layer deposited on blue-colored cellulose filter media (left), SEM images (middle) and optical microscopic images (right). With this blue-colored cellulose filter as a substrate, we can easily evaluate the nanofiber layer uniformity even without using SEM inspection.

\section{Filtration Efficiency and Dust Loading Test}

Fig. 4 shows the filter efficiency/loading test setup for nanofiber filters, which consists of an atomizer to generate test solution droplets, a diffusion dryer to convert the atomized droplets into solid particles, a neutralizer $\left({ }^{210} \mathrm{Po}\right)$ to discharge test particles, a test filter holder, and a Scanning Mobility Particle Sizer (SMPS 3936; TSI Inc.) to measure
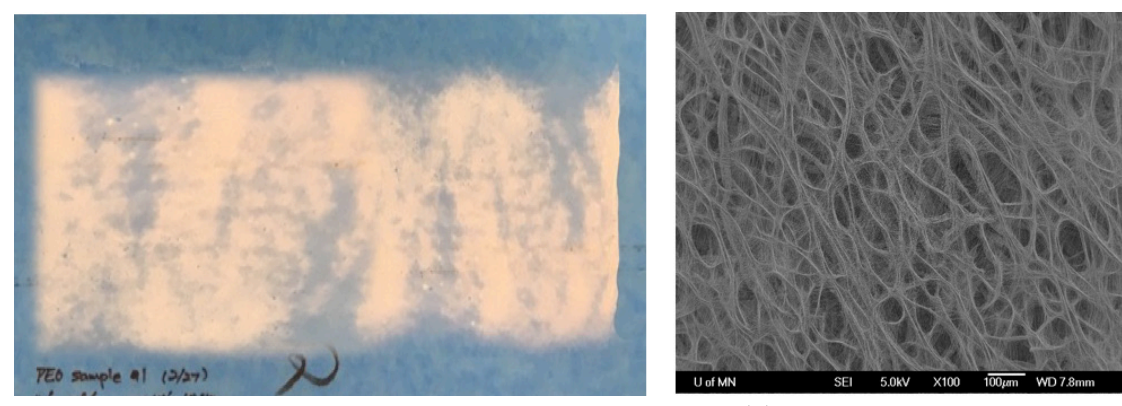

(a)
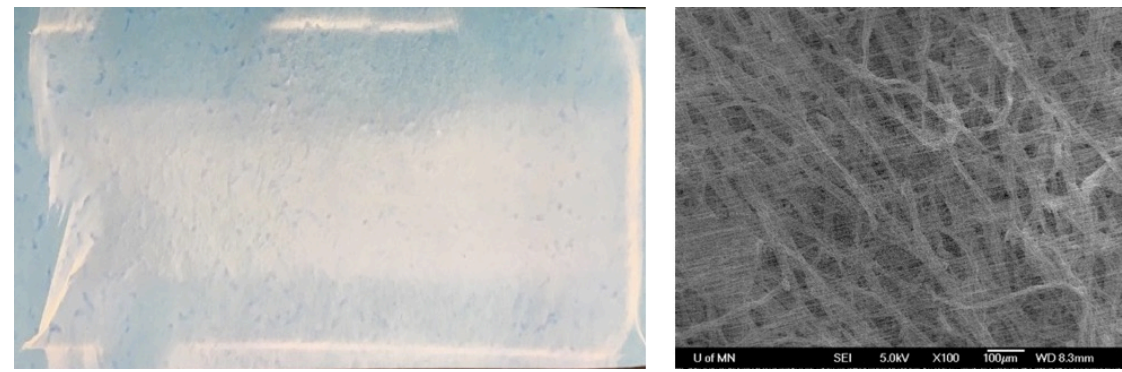

(b)
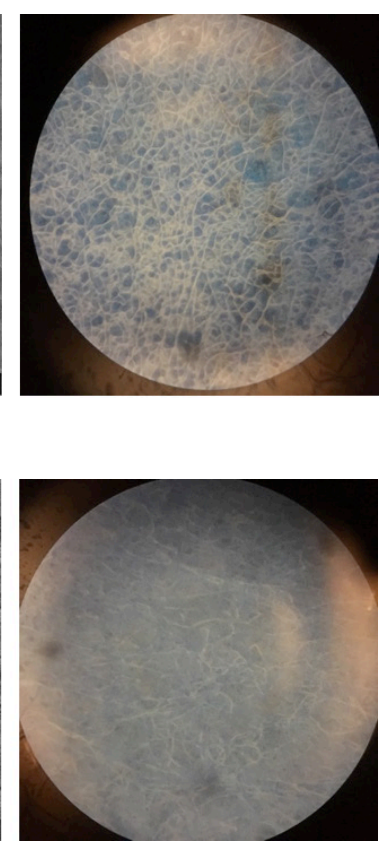

Fig. 3. (a) Non-uniform nanofiber deposition and (b) uniform nanofiber deposition by removing surface charge on the substrate filter media.

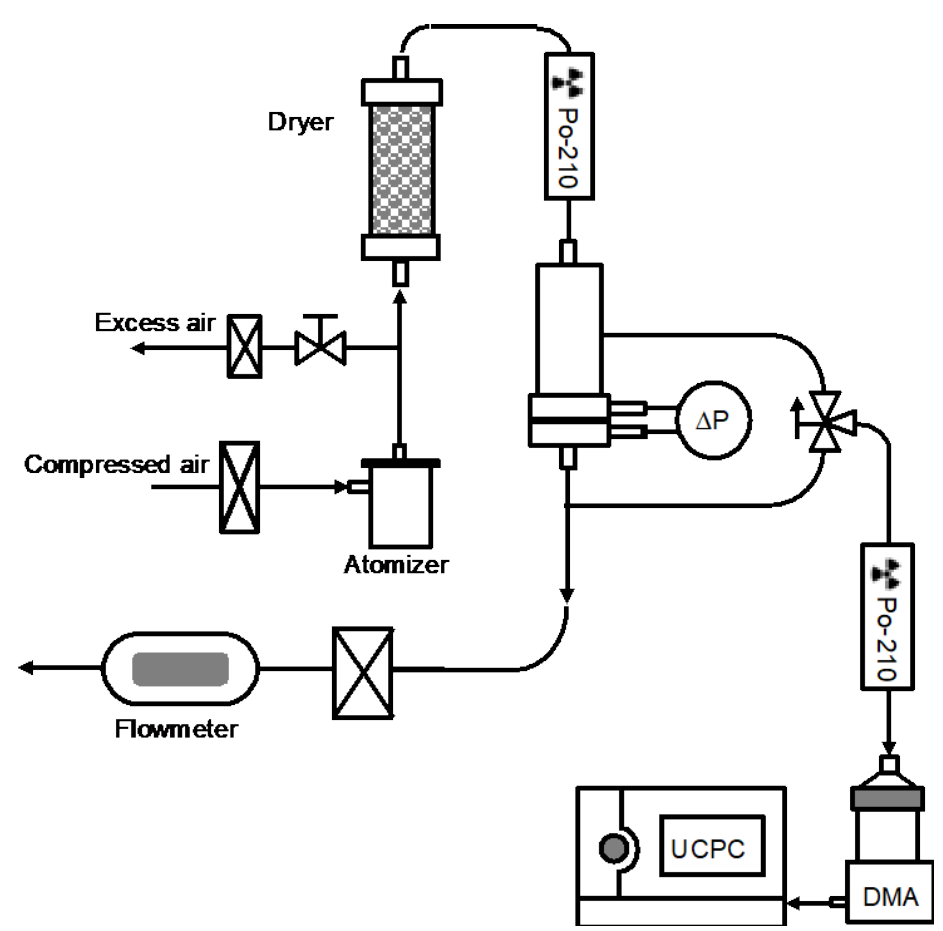

Fig. 4. Nanofiber filter efficiency/loading test setup. 
particle size distribution in upstream and downstream of the test filter. We generated $\mathrm{KCl}$ particles with an average particle diameter of $40 \mathrm{~nm}$ and the filtration face velocity was controlled to be $5 \mathrm{~cm} \mathrm{~s}^{-1}$. The filter pressure drop was monitored during the particle loading test by measuring differential pressure between upstream and downstream of the test filter. The filtration efficiency was calculated by measuring upstream and downstream particle size distributions from 20 to $500 \mathrm{~nm}$.

\section{RESULTS AND DISCUSSION}

\section{Nanofiber Layer Uniformity Effect on Filtration Performance}

Fig. 5 shows nanofiber layer uniformity effect on the filtration performance by comparing the filtration efficiencies between uniform nanofiber samples and non-uniform nanofiber samples with various initial pressure drops. Nanofibers were deposited on cellulose filter media and the initial pressure drop was controlled by changing the electrospinning time, which resulted in different nanofiber layer thickness. As shown in the filtration efficiency results (Fig. 5(a)), uniform nanofiber samples showed higher filtration efficiencies than non-uniform nanofiber samples with similar initial pressure drop. Comparing the uniform nanofiber sample with the pressure drop of $75.47 \mathrm{~Pa}$ to the non-uniform nanofiber sample with $82.45 \mathrm{~Pa}$, the uniform sample showed about $2 \%$ higher filtration efficiency than the non-uniform sample with higher pressure drop, which means thicker nanofiber layer.

Also, in order to quantify the overall filtration performance considering both the filtration efficiency and the pressure drop at the same time, figure of merit (FOM; also known as quality factor) was calculated for each filter sample as described below (Fig. 5(b)) (Wang et al., 2008).

$$
F O M=\frac{\ln (1 / P)}{\Delta P}
$$

where $P$ is the particle penetration (dimensionless) through the filter and $\Delta P$ is the pressure drop $(\mathrm{Pa})$ across the filter in the clean filter conditions. As implied from the equation, FOM is proportional to the filtration efficiency and reversely proportional to the pressure drop, thus higher FOM presents higher overall filtration performance. From the FOM comparison between the uniform and the non-uniform nanofiber samples, it was clearly observed that the uniform nanofiber samples presented higher FOM over the entire particle size range, which means uniform nanofiber deposition improved the overall filter performance in both filtration efficiency and pressure drop.

\section{Beaded Nanofibers and Branched Nanofibers}

Fig. 6(a) shows polyethylene oxide (PEO; M.W. 1,000,000; Alfa Aesar) nanofibers electrospun from polymer solutions with various viscosities. Given that the solution viscosity increases exponentially with the increase of the polymer concentration (Thomas and Thomas, 1960) and the polymer solution viscosity affects the nanofiber morphology, mainly fiber diameter and bead formation, we fabricated various shapes of nanofiber structures by changing the weight concentration of PEO solutions. In this research, we fabricated six different shapes of nanofiber media from PEO polymer solutions with various viscosities ranged from 29 to $362 \mathrm{mPa} \cdot \mathrm{s}$ (polymer weight concentrations ranged from $0.84 \%$ to $2 \%$ ).

The highest viscosity sample (Sample \#1, $362 \mathrm{mPa} \cdot \mathrm{s}$ ) showed smooth and straight nanofibers with an average fiber diameter of $172 \mathrm{~nm}$, and Sample \#2, the second highest (185 $\mathrm{mPa} \cdot \mathrm{s})$, generated elongated beads along the nanofibers with an average fiber diameter of $120 \mathrm{~nm}$. Sample \#3 (109 $\mathrm{mPa} \cdot \mathrm{s})$ showed very uniform and abundant elongated beads (bead thickness: around $500 \mathrm{~nm}$ ) along the nanofibers (average fiber diameter: $74 \mathrm{~nm})$ and Sample \#4 $(73 \mathrm{mPa} \cdot \mathrm{s})$ started to generate branched nanofibers, i.e., an additional nanofiber spinning out from the nanofiber bead, with an average fiber diameter of $62 \mathrm{~nm}$. Sample \#5 (45 mPa.s) showed a uniform branched nanofiber formation with average nanofiber

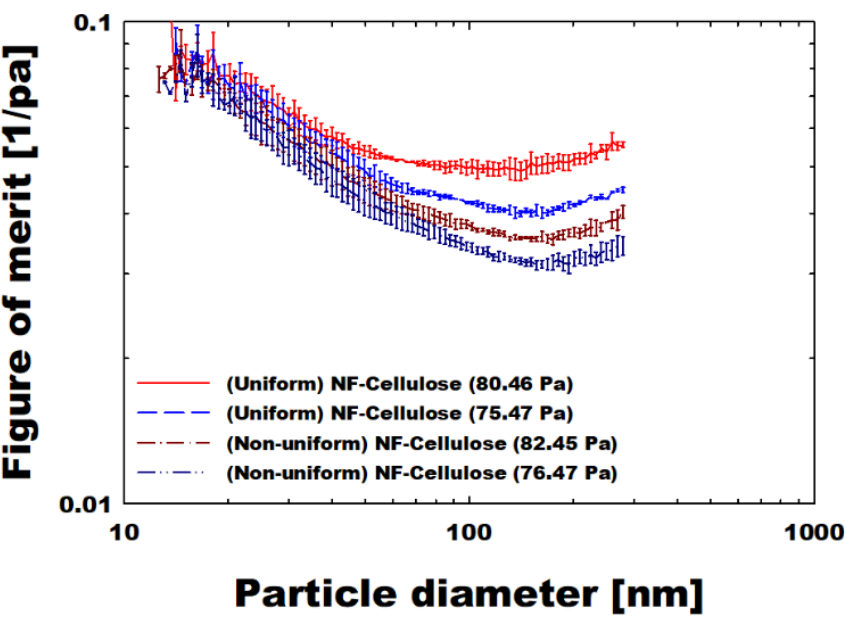

(b)

Fig. 5. Nanofiber layer uniformity effect on filtration performance: (a) filtration efficiency and (b) figure of merit. 
diameter of $45 \mathrm{~nm}$, and Sample \#6 (29 $\mathrm{mPa} \cdot \mathrm{s})$ started to show broken fibers between beads, thus it cannot be used as filter media.

Fig. 6(b) shows SEM images of branched nanofiber fabricated from polymer solution with a viscosity of $45 \mathrm{mPa} \cdot \mathrm{s}$ (Sample \#5), which has additional nanofibers spun out from each bead. These third nanofibers can improve the filtration efficiency dramatically as this filter has a much higher chance to capture particles while minimizing filter pressure drop owing to (1) the slip flow effect by the smaller fiber size (average diameter: $45 \mathrm{~nm}$ ) (Choi et al., 2017) and (2) the longer streamwise inter-fiber distance by the micron-sized beads.

In this research, three nanofiber filter samples (Sample \#1: smooth nanofiber; Sample \#3: elongated-bead nanofiber; and Sample \#5: branched nanofiber) were selected to evaluate the nanofiber morphology effect on the filtration performance. In order to compare the difference between the substrate filter media (cellulose filter) and three nanofiber filter samples in terms of filter loading, the pressure drop and the loaded particle mass were measured, respectively. To be specific, the pressure drop across each filter was measured by employing differential pressure gauge at every minute and the loaded particle mass was obtained by gravimetric method with the assumption of constant loading rate (Wang et al., 2016). The effects of pressure drop and particle loading according to four different filters were illustrated in Fig. 7. Since the nanofiber filter samples have additional nanofiber layers deposited on substrate filters, the nanofiber filter samples showed higher initial pressure drop (78.96, 84.69, and 90.42 Pa, respectively) than the substrate filter sample (66.51 Pa). However, pressure drop increase during the particle loading was observed to be much slower than that of substrate filter sample, thus the final dust holding capacity at $500 \mathrm{~Pa}$ was almost 10 times higher than the substrate filter sample. This result is due to the difference of particle loading mechanism between cellulose filters (Fig. 7(b), left) and nanofiber filters (Fig. 7(b), right), which

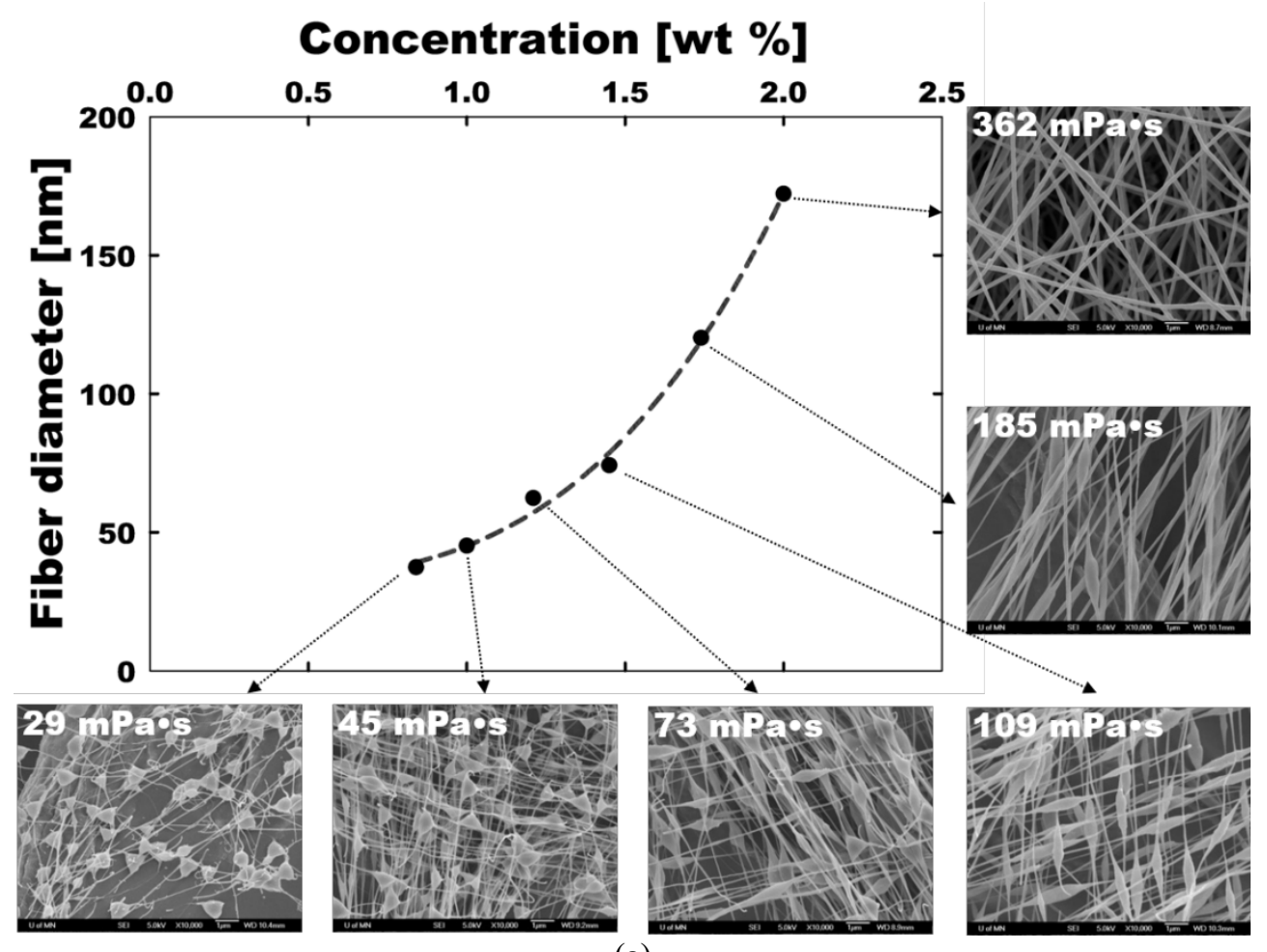

(a)
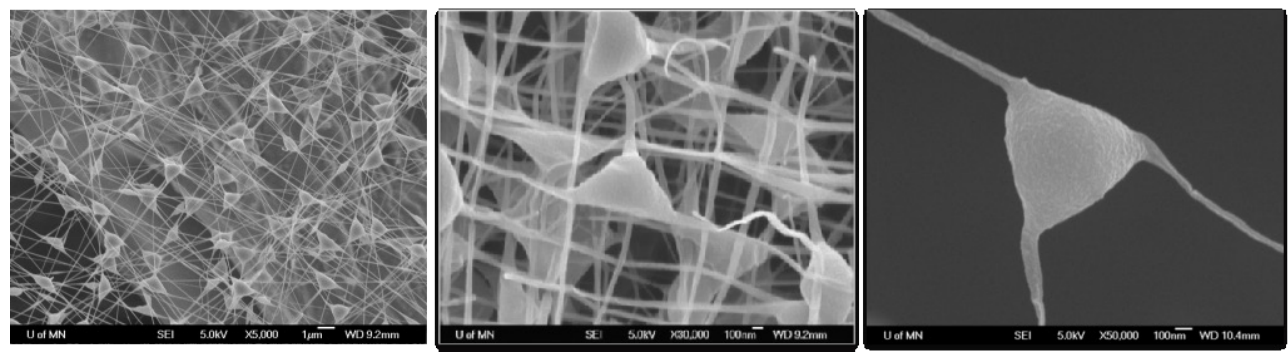

(b)

Fig. 6. PEO nanofiber formation with various polymer solution viscosities: (a) various shape of beaded nanofiber with the viscosity range from 29 to $362 \mathrm{mPa} \cdot \mathrm{s}$ and (b) SEM images of branched nanofiber. 


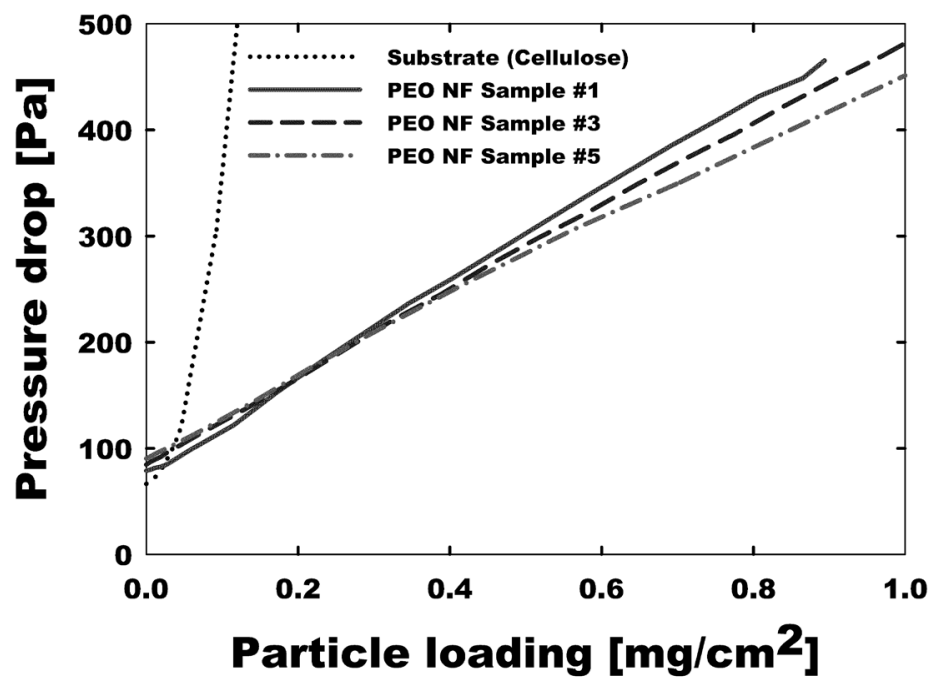

(a)

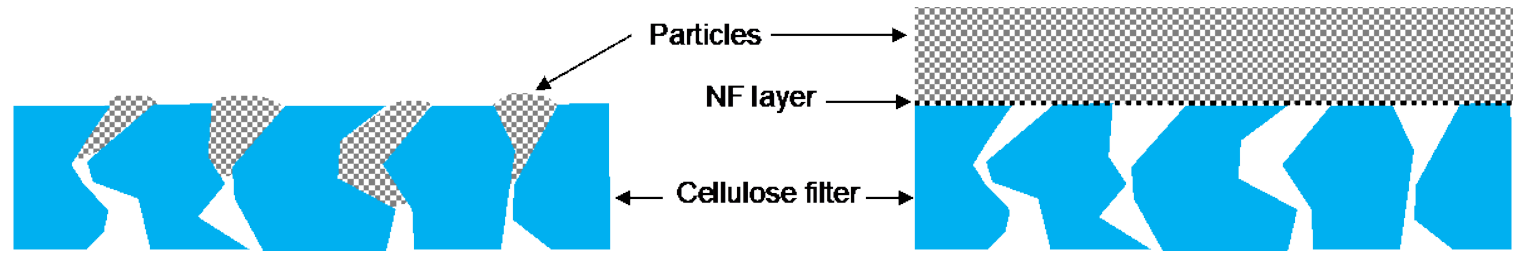

(b)
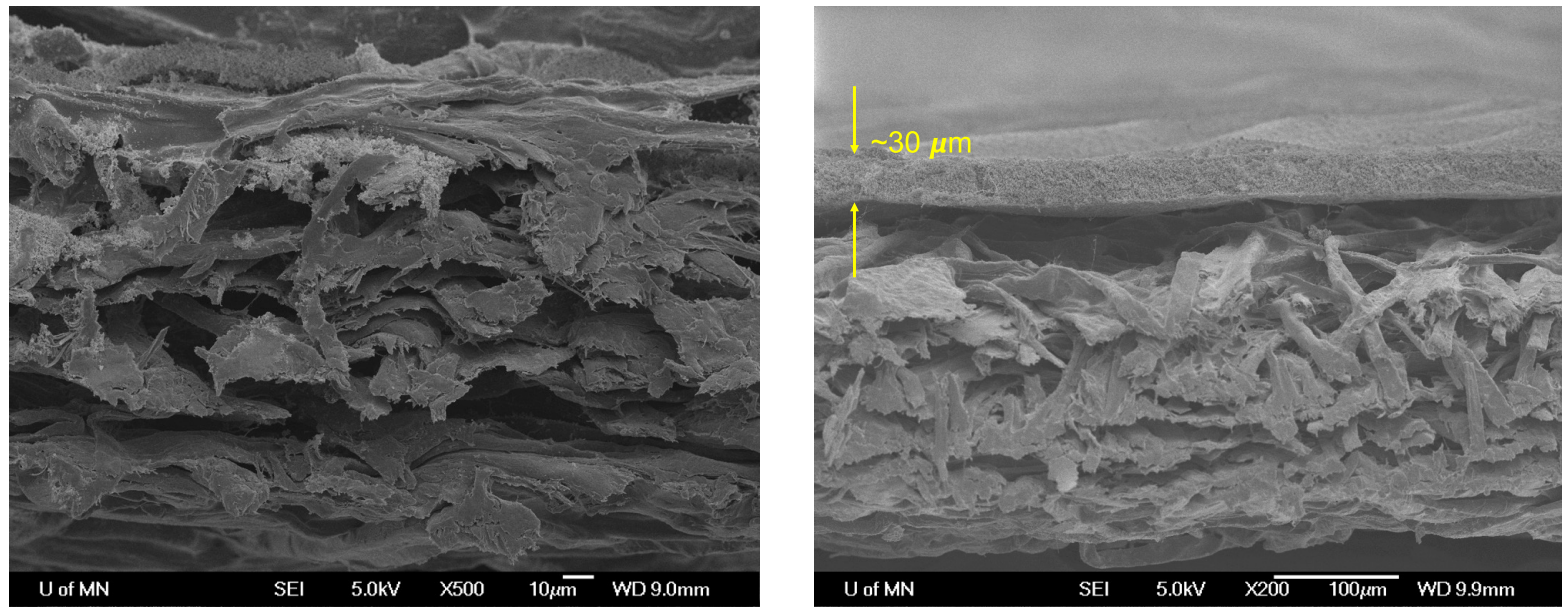

(c)

Fig. 7. Particle loading test comparison results between cellulose filter and nanofiber filters.

are called in-depth loading and surface loading, respectively. For cellulose filters (in-depth loading), the collected particles block the narrow air paths inside the filter media, which increases the pressure drop dramatically. However, for nanofiber filters (surface loading), the particles are collected on top of the nanofiber layer by sieving filtration mechanism and form a uniform dust cake all over the filtration surface. With this dust cake formation over the nanofiber layer, the air flow distributes very uniformly over the dust cake with low velocity and the air paths inside the filter media are still widely open, which result in the reduced air resistance across the filter media. Fig. 7(c) shows cross-sectional SEM images of cellulose filter (left) and nanofiber filter (right) after dust loading test at the final pressure drop of $500 \mathrm{~Pa}$. It was observed that air paths of the upstream side of the cellulose filter was clogged by the collected dust while nanofiber filter showed uniform dust cake (cake thickness: $30 \mu \mathrm{m}$ ) on the substrate filter and no significant amount of particles were observed inside the substrate filter air paths.

Fig. 8(a) shows filtration efficiency results of the substrate filter media and the nanofiber filter samples with a face velocity of $5 \mathrm{~cm} \mathrm{~s}^{-1}$ and nanofiber filter samples showed dramatic improvement in filtration efficiency comparing with the substrate filter media. For $100 \mathrm{~nm}$ particles, the filtration efficiency of substrate filter was $31.9 \%$, while the efficiencies of smooth NF (Sample \#1), elongated-bead NF 

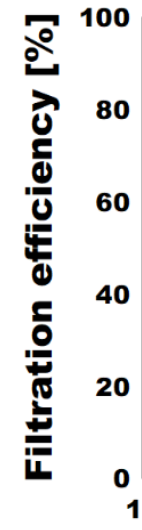

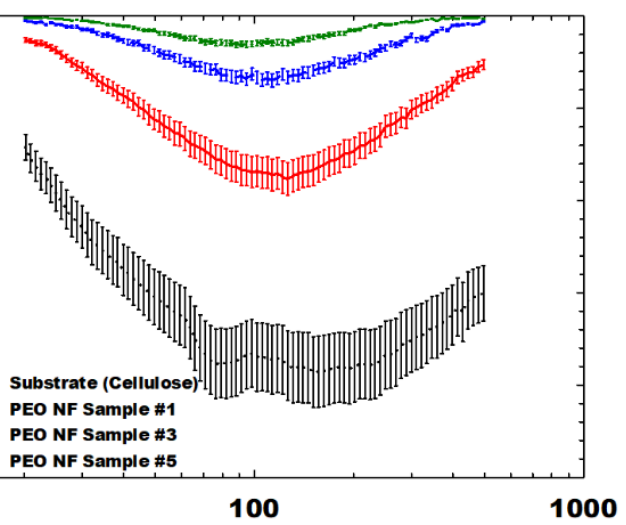

Particle diameter [nm]

(a)

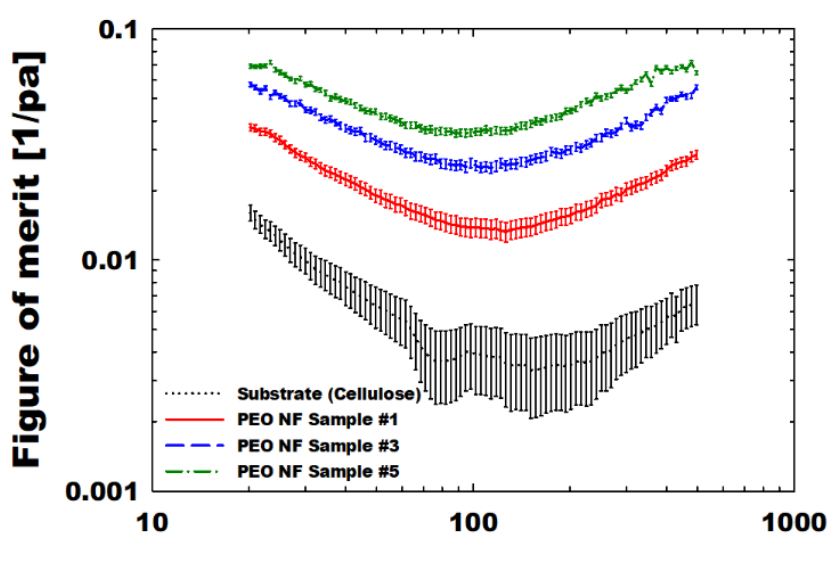

Particle diameter [nm]

(b)

Fig. 8. Filtration performance test for various beaded nanofiber filters: (a) filtration efficiency and (b) figure of merit.

(Sample \#3) and branched NF (Sample \#5) were measured $66.7 \%, 86.2 \%$ and $94.1 \%$, respectively. Beaded nanofiber filters (Sample \#3 and \#5) exhibited higher filtration efficiency than smooth nanofiber filter with the similar initial pressure drop due to their smaller fiber size and larger inter-fiber distance, which results in lower air resistance. Furthermore, branched NF sample (Sample \#5) showed even higher filtration efficiency due to the additional nanofibers spun out from each bead.

FOMs of substrate filter and nanofiber filters were calculated based on initial pressure drop and filtration efficiency test results in Fig. 8(b). As shown in the result, nanofiber filters exhibited much higher FOM than substrate filter media $\left(0.0058 \mathrm{~Pa}^{-1}\right.$ at $\left.100 \mathrm{~nm}\right)$ due to their higher filtration efficiencies despite of higher initial pressure drops. Especially, the branched nanofiber filter showed the highest FOM $\left(0.031 \mathrm{~Pa}^{-1}\right.$ at $\left.100 \mathrm{~nm}\right)$ over the elongated-bead nanofiber $\left(0.023 \mathrm{~Pa}^{-1}\right.$ at $\left.100 \mathrm{~nm}\right)$ and the smooth nanofiber filters $\left(0.014 \mathrm{~Pa}^{-1}\right.$ at $\left.100 \mathrm{~nm}\right)$.

\section{CONCLUSIONS}

We developed two kinds of high performance nanofiber filter media by increasing the uniformity of the nanofiber layer's deposition on the substrate and by fabricating branched nanofiber. These filters were compared with conventional filters with respect to particle removal efficiency and filter pressure drop, and the figures of merit confirmed their enhancement in performance. The uniformity of the nanofiber's deposition is a critical factor, as non-uniform deposition causes randomly distributed openings across the nanofiber layer, which, like pinholes, diminish the filtration efficiency. By removing the surface charge on the substrate media during electrospinning, the uniformity was drastically improved, resulting in a higher filtration efficiency with a lower pressure drop. We also fabricated branched nanofiber by adjusting the viscosity of the polymer solution to produce additional fibers radiating from every single bead on the main fibers. The performance of the nanofiber filters was dramatically improved by the decrease in air flow resistance, which resulted from (1) a longer distance streamwise between the fibers and (2) a smaller diameter for the fibers. The enhanced efficiency was also due to (3) the additional fibers branching out from the main fibers.

\section{ACKNOWLEDGMENTS}

The authors thank the support of members of the Center for Filtration Research: 3M Corporation; A.O. Smith Company; Applied Materials, Inc.; BASF Corporation; Boeing Company; Corning Co.; China Yancheng Environmental Protection Science and Technology City; Cummins Filtration Inc.; Donaldson Company, Inc.; Entegris, Inc.; Ford Motor Company; Guangxi Wat Yuan Filtration System Co., Ltd; LG Electronics Co.; MSP Corporation; Samsung Electronics Co., Ltd.; Xinxiang Shengda Filtration Technology Co., Ltd.; TSI Inc.; W. L. Gore \& Associates, Inc.; Shigematsu Works Co., Ltd.; and the affiliate member National Institute for Occupational Safety and Health (NIOSH).

\section{REFERENCES}

Barhate, R.S., Loong, C.K. and Ramakrishna, S. (2006). Preparation and characterization of nanofibrous filtering media. J. Membr. Sci. 283: 209-218.

Barhate, R.S. and Ramakrishna, S. (2007). Nanofibrous filtering media: Filtration problems and solutions from tiny materials. J. Membr. Sci. 296: 1-8.

Baumgarten, P.K. (1971). Electrostatic spinning of acrylic microfibers. J. Colloid Interface Sci. 36: 71-79.

Choi, H.J., Kumita, M., Seto, T., Inui, Y., Bao, L., Fujimoto, T. and Otani, Y. (2017). Effect of slip flow on pressure drop of nanofiber filters. J. Aerosol Sci. 114: 244-249.

Drew, C., Wang, X., Samuelson, L.A. and Kumar, J. (2003). The effect of viscosity and filler on electrospun fiber morphology. J. Macromol. Sci. Part A 40: 1415-1422.

Fong, H., Chun, I. and Reneker, D. (1999). Beaded nanofibers formed during electrospinning. Polymer 40: 4585-4592.

Gao, H., Yang, Y., Akampumuza, O., Hou, J., Zhang, H. and 
Qin, X. (2017). A low filtration resistance threedimensional composite membrane fabricated via free surface electrospinning for effective $\mathrm{PM}_{2.5}$ capture. Environ. Sci. Nano 4: 864-875.

Hekmati, A.H., Rashidi, A., Ghazisaeidi, R. and Drean, J.Y. (2013). Effect of needle length, electrospinning distance, and solution concentration on morphological properties of polyamide-6 electrospun nanowebs. Text. Res. J. 83: 1452-1466.

Huang, Z., Zhang, Y., Kotaki, M. and Ramakrishna, S. (2003). A review on polymer nanofibers by electrospinning and their applications in nanocomposites. Compos. Sci. Technol. 63: 2223-2253.

Leung, W.W.F., Hung, C.H. and Yuen, P.T. (2009). Experimental investigation on continuous filtration of sub-micron aerosol by filter composed of dual-layers including a nanofiber layer. Aerosol Sci. Technol. 43: 1174-1183.

McKee, M.G., Wilkes, G.L., Colby, R.H. and Long, T.E. (2004). Correlations of solution rheology with electrospun fiber formation of linear and branched polyesters. Macromolecules 37: 1760-1767.

Nel, A. (2005). Air Pollution-related illness: Effects of particles. Science 308: 804-806.

Oberdörster, G., Stone, V. and Donaldson, K. (2007). Toxicology of nanoparticles: A historical perspective. Nanotoxicology 1: 2-25.

Shenoy, S.L., Bates, W.D., Frisch, H.L. and Wnek, G.E. (2005). Role of chain entanglements on fiber formation during electrospinning of polymer solutions: Good solvent, non-specific polymer-polymer interaction limit. Polymer 46: 3372-3384.
Stone, V. and Donaldson, K. (2006). Signs of stress. Nat. Nanotechnol. 1: 23-24.

Thomas, D.K. and Thomas, T.A.J. (1960). Viscosityconcentration relationships in solutions of high polymers. J. Appl. Polym. Sci. 3: 129-131.

Wang, J., Kim, S.C. and Pui, D.Y.H. (2008). Investigation of the figure of merit for filters with a single nanofiber layer on a substrate. J. Aerosol Sci. 39: 323-334.

Wang, Q., Lin, X. and Chen, D.R. (2016). Effect of dust loading rate on the loading characteristics of high efficiency filter media. Powder Technol. 287: 20-28.

Wang, Y., Wang, G., Chen, L., Li, H., Yin, T., Wang, B., Lee, J.C.M. and Yu, Q. (2009). Electrospun nanofiber meshes with tailored architectures and patterns as potential tissue-engineering scaffolds. Biofabrication, 1: 015001 .

Wang, Z., Zhao, C. and Pan, Z. (2015). Porous bead-onstring poly(lactic acid) fibrous membranes for air filtration. J. Colloid Interface Sci. 441: 121-129.

Xu, J., Liu, C., Hsu, P.C., Liu, K., Zhang, R., Liu, Y. and Cui, Y. (2016). Roll-to-roll transfer of electrospun Nanofiber film for high-efficiency transparent air filter. Nano Lett. 16: 1270-1275.

Yun, K.M., Suryamas, A.B., Iskandar, F., Bao, L., Niinuma, H. and Okuyama, K. (2010). Morphology optimization of polymer nanofiber for applications in aerosol particle filtration. Sep. Purif. Technol. 75: 340-345.

Received for review, July 12, 2019 Revised, September 24, 2019 Accepted, October 31, 2019 